\title{
Analyzing and Improving the Robustness of Tabular Classifiers using Counterfactual Explanations
}

\author{
$1^{\text {st }}$ Peyman Rasouli \\ Department of Informatics \\ University of Oslo \\ Oslo, Norway \\ peymanra@ifi.uio.no
}

\author{
$2^{\text {nd }}$ Ingrid Chieh $\mathrm{Yu}$ \\ Department of Informatics \\ University of Oslo \\ Oslo, Norway \\ ingridcy@ifi.uio.no
}

\begin{abstract}
Recent studies have revealed that Machine Learning (ML) models are vulnerable to adversarial perturbations. Such perturbations can be intentionally or accidentally added to the original inputs, evading the classifier's behavior to misclassify the crafted samples. A widely-used solution is to retrain the model using data points generated by various attack strategies. However, this creates a classifier robust to some particular evasions and can not defend unknown or universal perturbations. Counterfactual explanations are a specific class of post-hoc explanation methods that provide minimal modification to the input features in order to obtain a particular outcome from the model. In addition to the resemblance of counterfactual explanations to the universal perturbations, the possibility of generating instances from specific classes makes such approaches suitable for analyzing and improving the model's robustness. Rather than explaining the model's decisions in the deployment phase, we utilize the distance information obtained from counterfactuals and propose novel metrics to analyze the robustness of tabular classifiers. Further, we introduce a decision boundary modification approach using customized counterfactual data points to improve the robustness of the models without compromising their accuracy. Our framework addresses the robustness of black-box classifiers in the tabular setting, which is considered an under-explored research area. Through several experiments and evaluations, we demonstrate the efficacy of our approach in analyzing and improving the robustness of black-box tabular classifiers.
\end{abstract}

Index Terms-Robustness, Black-box Models, Tabular Data, Counterfactual Explanations, Decision Boundary

\section{INTRODUCTION}

Machine Learning (ML) techniques, especially deep learning models, are rapidly progressing as they demonstrate promising results in various applications. Nevertheless, the concern around their safety and reliability has limited their adoption for safety-critical use-cases. Recent studies have revealed the vulnerability of ML models towards adversarial and non-adversarial attacks [1]. Precisely, an attack is a data point resulted from (either intentional or accidental) imperceptible perturbations added to an original instance that can mislead the model's decision-making. As a result, the model makes misprediction for samples whose associated class is obvious from the expert's viewpoint. This problem has created a severe barrier to the applicability of machine learning techniques in high-stake applications and thereof inspired the research community to investigate the robustness of current models.
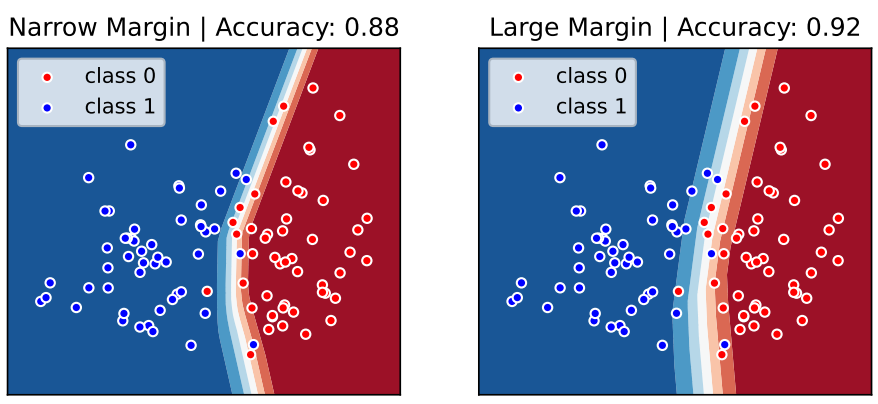

Fig. 1: Visualization of class margin: narrow vs. large.

There exist several works around creating model- and domain-specific attacks for the image domain [2]. The primary goal of such techniques is to evaluate the robustness of a model concerning invisible noises. Alongside the accuracy evaluation, robustness analysis can help a developer to pick the less vulnerable model to adversarial perturbations. There are numerous research works for improving the robustness of ML models [3]. Retraining a model using adversarial samples generated by various methods is considered a common procedure. Although this is a practical approach for robustifying the model against specific threats, it may be ineffective in tackling unknown and non-adversarial perturbations occurring in realworld applications [4].

Ideally, we desire a model that is robust against arbitrary modified inputs using unknown perturbations. A possible solution is creating a large margin classifier. The notion of margin, the minimum distance of data points to the decision boundary, has been shown theoretically and empirically effective in creating robust and accurate models [5]. In other words, having large margins between the decision space of different classes makes the small and imperceptible perturbations ineffective in manipulating the model's decision-making. To better illustrate the importance of large margins, we refer to Figure 1 It shows two black-box models with a narrow margin (left) and a large margin (right) on the same data set. In addition to higher predictive performance, large margin classifiers are proven to be more robust against adversarial/non-adversarial perturbations [6]. The wider the margin is, the more effort (i.e., more perturbation) is needed to create an instance that 
crosses the decision boundary. Consequently, increasing the class margin results in enhancing the robustness of the model against universal perturbations.

Mainly, there are two ways to increase the class margin of a classifier: changing the loss function [7], [8] and retraining the model with boundary-shifting samples [5], [9]. Unfortunately, methods falling in these categories are either applicable to white-box deep neural networks or designed for the image domain. This prevents their adoption for the robustness investigation of tabular classifiers, which is a widely used setting in many industrial use-cases [10]. Moreover, requiring access to the model internals can diminish the applicability of current methods for limited access scenarios and constrain their adoption for competent, black-box models such as Random Forests (RF) and Support Vector Machines (SVM).

In this work, we propose a framework based on Counterfactual Explanations (CE) to address two main challenges: 1) analyzing the robustness of black-box models created for tabular data and 2) improving the robustness of the models by increasing the class margins. Counterfactual explanations are a specific class of post-hoc explanation methods that provide minimal modification to the input features in order to obtain a particular outcome from the model [11]. There is a remarkable similarity between counterfactual explanations and the universal perturbations as they both deceive the model's behavior with unknown and arbitrary changes to the input. Meanwhile, counterfactual explanation methods can generate instances from specific classes, making them suitable for analyzing or improving the robustness of a classifier with respect to distinct (minority) classes. For robustness analysis, we generate unconstrained counterfactuals that can be located anywhere on the decision surface of the targeted class. We introduce overall and class-wise robustness metrics based on the distance between the original samples and corresponding counterfactuals. To improve the robustness of a classifier concerning a particular class, we generate counterfactuals on the decision boundary of the neighborhood classes and retrain the model with a representative set of the crafted samples to enlarge the class margins. We propose a multi-objective explanation approach to define required objective functions for unconstrained and boundary counterfactual generation. Our optimization scheme does not require access to the model's internal, making the framework model-agnostic. The primary contributions of the work can be summarized as follows:

- We devise a multi-objective and model-agnostic explanation method that enables formulating unconstrained and boundary counterfactuals and applies to any black-box model in tabular settings.

- We study the utility of unconstrained counterfactuals in generating the universal perturbations and propose overall and class-wise robustness analysis metrics based on their distance information.

- We propose a novel approach for enlarging class margins using boundary counterfactuals to improve the robustness of the model against universal perturbation.

- We demonstrate the efficacy of our framework through extensive evaluations and comparisons with state-ofthe-art and provide an open-source benchmark for the research community: https://github.com/peymanrasouli/ RobustML

The rest of the paper is organized as follows: Section II provides a literature review of various evasion and defense strategies; Section III introduces our counterfactual explanation-based framework for analyzing and improving the robustness of black-box tabular classifiers; Section IV presents and discusses the conducted experiments towards evaluating the efficacy of our proposed method; at last, Section $\mathrm{V}$ concludes the paper and states the future works.

\section{RELATED WORKS}

Tabular Domain: In [10], authors propose a novel approach to modify and adapt well-known attack strategies in the image domain for the tabular data. They mainly focus on the imbalance data set scenarios, Extreme Gradient Boosting models, representation of data types and feature values, and the modification of non-editable information. In [12], authors present LowProFool, an imperceptible adversarial attack for tabular data. Their method is designed for withe-box deep neural networks, meaning that the model's loss function is accessible. In contrast to common adversarial attacks that arbitrarily perturb input features to fool the model, the proposed method modifies less important features from the expert's perspective. The authors encode expert knowledge as a feature importance vector and integrate it with the original loss function.

Decision Space: In [4], authors study the robustness of deep neural networks for the image domain with respect to unrestricted evasion attacks. Specifically, they analyze the model's behavior concerning class margins and improve the model's robustness by increasing the proportion of samples in vulnerable classes. In [13], authors introduce a novel attack called OptMargin, which generates adversarial examples robust to small perturbations. Moreover, they analyze the neighborhood around input instances by looking at properties of surrounding decision boundaries, namely the distances to the boundaries and the adjacent classes.

Class Margin: In [9], authors introduce a new training approach called Increasing Margin Adversarial (IMA) to improve the robustness of deep neural networks for the image domain. During training, the IMA method increases the margins of training samples by moving the decision boundaries of the DNN model far away from the training samples to improve robustness. In [5], authors propose a novel loss function to enlarge the class margins of withe-box DNNs. What makes the loss function unique compared to other existing approaches is its ability to impose a margin on any chosen set of layers (including input and hidden layers). The devised loss function is general and can be complementary to existing data augmentation and regularization techniques.

Loss Function: In [7], authors improve the robustness of white-box DNNs by introducing a novel adversarial training utilizing Triplet Loss, a popular technique of Distance Metric Learning. The training approach enlarges the distance 
between adversarial and normal examples in the embedding space, resulting in model robustness. In [8], authors present DeepFool, an attack generation algorithm for white-box deep neural networks. Based on the iterative linearization of the classifier, it generates minimal perturbations that change the original prediction of the input. The authors employ DeepFool to evaluate and compare the robustness of different classifiers in the vision domain. Besides, they improve the robustness of a classifier by augmenting training data with adversarial samples created by DeepFool.

Takeaways: In reviewing the literature, we observed several under-explored areas that are motivating this work:

- The majority of the works are dedicated to the image field [2]. However, the tabular domain as a popular setting in many industrial use-cases has been remained underexplored. Thus, more research towards creating efficient, universal perturbations and practical approaches for improving the robustness of tabular classifiers is required.

- Most of the existing methods for tabular data merely analyze the robustness of white-box deep neural networks [10]. It means, to evaluate the performance, access to the model's internals is required. In fact, this can diminish the applicability of current methods in limited access scenarios (e.g., accessing the model using an API). Moreover, the specificity of such techniques constrains their adoption for competent models like RFs and SVMs and restricts practical third-party assessments.

- The existing attack strategies for tabular domain [12] specifically target the scenarios where the attacker aims to manipulate the model's behavior intentionally. Therefore, a validated/upgraded model via such techniques may fail concerning universal, unknown perturbations that may occur in real-world applications.

- Compared to the image field, where there exist various approaches for enhancing the robustness, such as altering the decision boundary [5], [9] and modifying the loss function [7], there is a lack of similar frameworks for black-box ML models in the tabular domain. Thus, having a systematic framework for creating robust tabular classifiers with a desirable degree of accuracy is a must.

\section{Counterfactual Explanations FOR Model ROBUSTNESS}

Counterfactual explanations are a specific class of post-hoc explanation methods that explain how to modify the input in order to obtain a particular outcome from the model. The fundamental definition implies that the changes need to be minimal and can be applied to arbitrary features, resulting in an instance similar to the original one. The analogy of counterfactuals with universal perturbations motivates us to study their utility in analyzing and improving the robustness of the models. In the following sections, first, we introduce a multi-objective and model-agnostic explanation method to generate unconstrained and boundary counterfactual instances for tabular classifiers. Second, we propose a robustness evaluation approach to measure the overall and class-wise robustness of the models. Third, we devise a procedure to improve the robustness of the models via modifying their class margins.

\section{A. Multi-objective Counterfactual Explanation Method}

In this section, we propose a multi-objective counterfactual explanation method to generate unconstrained and boundary data points that are being used in robustness analysis and improvement algorithms, respectively. Formally, a counterfactual explanation generates a perturbation vector for the input features to create a new instance that results in an outcome specified by the user. Consider a tabular data set $D=\left(X^{m}, Y^{C}\right)$, where $X^{m}$ is an $m$ dimensional feature space and $Y$ is a target set containing $C$ classes. We split the data into train $D_{\text {train }} \in D$ and test set $D_{\text {test }} \in D$. Let $f$ be a black-box model trained on $D_{\text {train }}$ that maps inputs to the targets, i.e., $f: X^{m} \rightarrow Y^{C}$. For a given input $(x, y) \in D_{\text {test }}$ with $f(x)=y$ and a desired outcome $y^{\prime} \in C, y^{\prime} \neq y$, our goal is to find a counterfactual $x^{\prime}$ as close to $x$ as possible such that $f\left(x^{\prime}\right)=y^{\prime}$. We formulate this problem in a model-agnostic and multi-objective optimization framework that allows imposing arbitrary requirements without demanding access to the model's internals or loss function. We use Non-dominated Sorting Genetic Algorithm (NSGA-III) [14], an evolutionary multi-objective optimization approach, to find the optimal solution. It generates multiple and diverse counterfactuals for a given input in a reasonable amount of time which is wellsuited for the robustness improvement phase, where we need to create counterfactuals for a large number of training data points. The algorithm can also handle mixed-feature data sets, including numerical and categorical features, without additional processing. We formulated the different desiderata for generating counterfactual instances using three objective functions $O_{\text {label }}, O_{\text {probability }}$, and $O_{\text {distance }}$ described below.

1) $O_{\text {label }}$ : This function ensures the generated sample belongs to the desired class. We define the labeling cost for a potential counterfactual $x^{\prime}$ and the desired class $c$ as follows:

$$
O_{\text {label }}\left(x^{\prime}, c\right)= \begin{cases}0, & \text { if } f\left(x^{\prime}\right)=c \\ 1, & \text { otherwise }\end{cases}
$$

the function returns 0 if the desired label is achieved, otherwise, it outputs 1 .

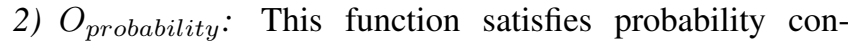
straints for the generated counterfactuals. We define two variations $O_{\text {probability }}^{\text {unconstrained }}$ and $O_{\text {probability }}^{\text {boundary }}$ that are used for generating unconstrained and boundary-located counterfactuals, respectively. The $O_{\text {probability }}^{\text {unconstrained }}$ is defined as follows:

$$
O_{\text {probability }}^{\text {unconstrained }}\left(x^{\prime}, c, p\right)=\max \left(0,\left(p-f_{c}\left(x^{\prime}\right)\right)\right)
$$

where $f_{c}\left(x^{\prime}\right)$ is the prediction probability of $x^{\prime}$ for the desired class $c$ and $p$ is a probability threshold. This function treats every counterfactual above the threshold $p$ as equal (zero cost). The defined objective is suitable for analyzing the robustness of a model with respect to different levels of confidence. Given an original input $x$, we generate an on-boundary counterfactual $x^{\prime}$ for the desired class $c$ using the following function:

$$
O_{\text {probability }}^{\text {boundary }}\left(x, x^{\prime}, c\right)=\left|f(x)-f_{c}\left(x^{\prime}\right)\right|
$$


The function measures the absolute difference between the probability of the original input $x$ and the potential counterfactual $x^{\prime}$. By minimizing the difference, we create a data point located on the margin of the classes.

3) $O_{\text {distance: }}$ This function calculates the distance between an original input $x$ and a counterfactual instance $x^{\prime}$ using a norm appropriate to the data:

$$
O_{\text {distance }}\left(x, x^{\prime}\right)=\left\|x-x^{\prime}\right\|
$$

The optimization algorithm creates optimal counterfactual instances by minimizing the defined objectives. To this end, we create two combinations of the objective functions to handle the different data point requirements for robustness analysis and robustness improvement. We construct UnconstrainedCF combination that generates samples located in the decision surface of the desired class:

$$
\text { UnconstrainedCF }=\left\{O_{\text {label }}, O_{\text {probability }}^{\text {unconstrained }}, O_{\text {distance }}\right\}
$$

This combination is equivalent to universal perturbation generation because it mainly focuses on creating counterfactuals located in the input's minimum distance. We can analyze the robustness of a model by measuring the distance between the original instances and the corresponding unconstrained counterfactuals. In Section III-B, we propose useful metrics for robustness analysis based on this information. We create BoundaryCF combination that generates samples on the boundary of two adjacent classes:

$$
\text { BoundaryCF }=\left\{O_{\text {label }}, O_{\text {probability }}^{\text {boundary }}, O_{\text {distance }}\right\}
$$

The created samples are utilized for calculating the precise distance of the original data points to the decision boundary. We leverage this information in Section III-C to increase the class margins of the classifier, resulting in a robust model against universal perturbations. A few examples of unconstrained and boundary counterfactuals are depicted in Figure 2 .

\section{B. Robustness Analysis using CE}

This section proposes an algorithm to evaluate the robustness of black-box ML models in the tabular setting. We provide overall and class-wise robustness metrics based on the distance information derived from unconstrained counterfactual instances. Our metrics are model-agnostic; therefore, we can use them to compare the performance of various models for an existing application. Furthermore, we can identify the vulnerable classes using the class-wise robustness metric that can be subjected to robustness improvement later.

Algorithm 1 outlines our devised approach for analyzing the robustness of classifier $f$ with respect to the test data $D_{\text {test }}$. It comprises of two procedures COUNTERFACTUALGENERATION and RoBUSTnESSMEASURMENT that generate counterfactual instances and measure the robustness of the model based on the generated samples, respectively. We generate counterfactuals of every classes $\forall j \in C$ for all samples in the test set $\forall x \in D_{\text {test }}$, then measure their distance to the original samples (denoted by $D$ ) as well as count the number of the created counterfactuals (denoted by $N$ ).

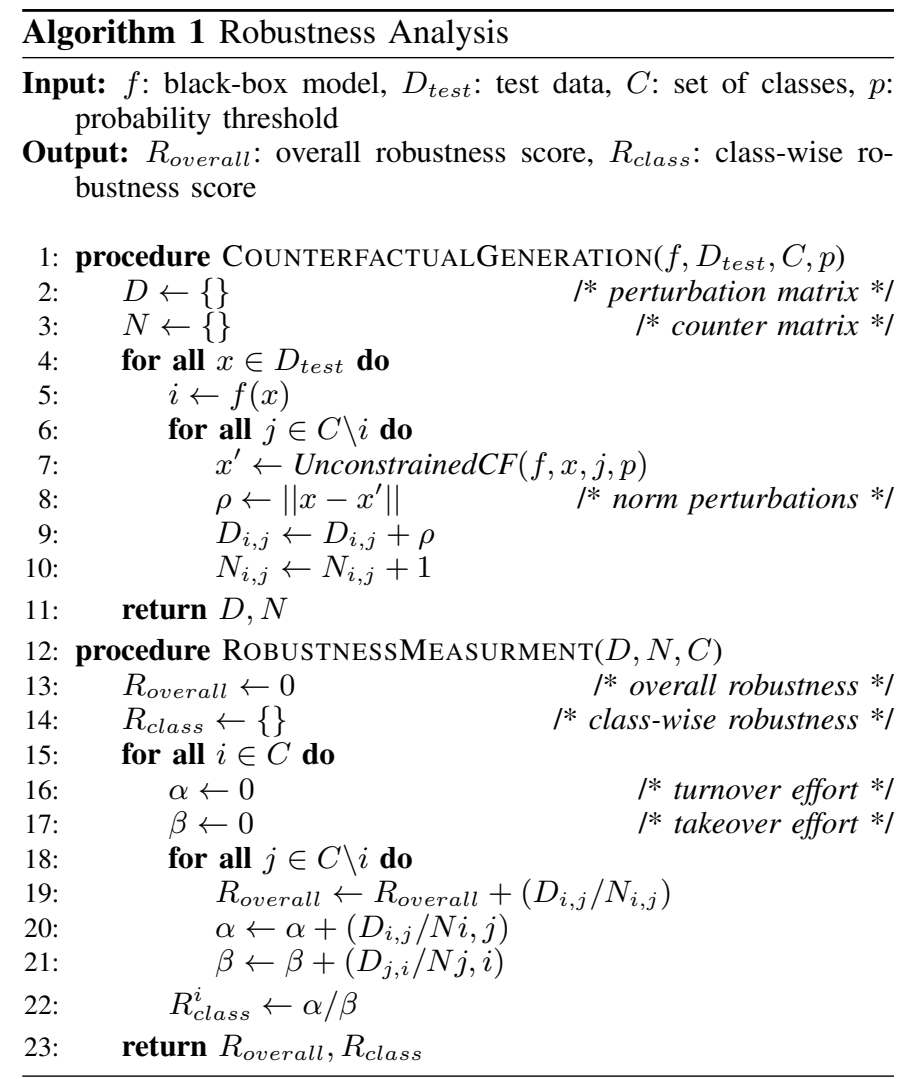

Afterward, we measure the overall and class-wise robustness of the model. The overall robustness, i.e., $R_{\text {overall }}$, is the summation of all normalized distances between all pairs of classes $\{i, j\} \in C, i \neq j$. The larger the $R_{\text {overall }}$ is, the more effort is needed to generate adversarial samples that cross the decision boundary and deceive the model $f$. This is a proper metric for comparing different models trained on the same data set that allows the expert to select the most robust one. The class-wise robustness, i.e., $R_{\text {class }}$, provides a comprehensive insight into the decision boundaries between distinct classes. For every class $\forall j \in C$, it calculates the ratio between the turnover and takeover effort of the class. For a specific class $i \in C$, the turnover effort $(\alpha)$ is the amount of required perturbations to change its associated samples to other classes, while the takeover effort $(\beta)$ is the portion of needed perturbations for the samples of other classes to be classified as $i$. An $R_{\text {class }}$ value greater than 1.0 refers to a robust class, whereas less than 1.0 indicates a vulnerable class. Ideally, a globally robust classifier has an $R_{\text {class }}^{i} \simeq 1.0, \forall i \in C$, implying equal margins between the classes. This is a useful metric to identify the model's shortcoming concerning specific classes. Based on this information, a developer can take necessary actions such as data gathering and class balance to enhance the class-wise robustness.

\section{Robustness Improvement using CE}

The idea of enlarging class margins in the decision boundary has been theoretically and empirically proven to be effective in increasing the robustness of the model [6]. Inspired by this, we 


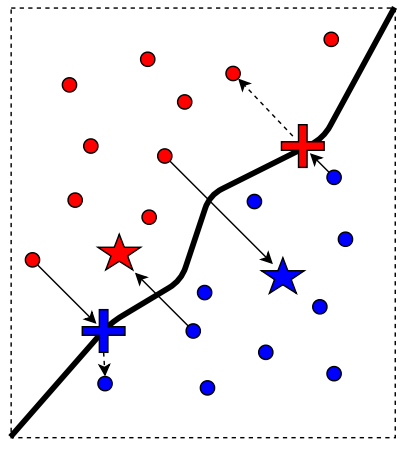

- Original data point - class 0

- Original data point - class 1

岤 Unconstrained CF - class 0

Unconstrained CF - class 1

Boundary CF - class 0
Boundary CF - class 1

$\longrightarrow$ Distance $d_{x \rightarrow x^{\prime}}$

.... Distance $d_{x^{\prime} \rightarrow x_{\text {groundtruth }}^{\prime}}$

Fig. 2: Illustration of boundary vs. unconstrained CFs.

propose a method to increase the class margins of any blackbox classifier without diminishing its accuracy. We generate counterfactuals on the decision boundaries for correctly predicted samples and use them to modify and enlarge the class margins. A large margin classifier takes more effort (i.e., larger and perceptible perturbations to the inputs) to be confused. Therefore, it is considered as a practical strategy towards protecting against intentional/accidental evasive perturbations. Precisely, a vulnerable class suffers from a narrow margin with the decision boundary. Therefore, we enlarge the margin in favor of the class to improve its robustness. This procedure can be repeated for every desired class until a globally robust model with a balanced margin for every class is achieved.

Consider a black-box $f$ created on the training data $D_{\text {train }}$ that is vulnerable to class $c \in C$. Let us denote to $D_{\text {correct }} \subseteq$ $D_{\text {train }}$ and $D_{\text {correct }}^{c} \subset D_{\text {correct }}$ as the correctly predicted training data by the model $f$ for all classes and for class $c$, respectively. Our goal is to generate counterfactuals located on the decision boundaries for $D_{\text {correct }}^{c}$ and retrain the model $f$ with a representative set of the generated samples to enlarge the margins of the class. The two tasks, i.e., generating boundary counterfactuals and selecting appropriate samples, play crucial roles in properly increasing the robustness of the model. Compared to unconstrained counterfactuals, which can be located anywhere in the decision space of the target class, a boundary counterfactual provides the precise distance of the original instance to the decision boundary. We illustrated these two types of counterfactuals in Figure 2. Moreover, it should be noted that there may be classes with overlapping or similar distributions that make the boundary shifting difficult. Instead of simply retraining the model using a portion of or the entire crafted examples [4], here, we propose a distance-based metric for selecting valid data points. It is a reliable metric as it helps us to fine-tune the behavior of a specific class without interfering with the performance of the adjacent classes.

Algorithm 2 summarizes our devised approach for improving the robustness of the classifier $f$. We use CounterFACTUALGENERATION to generate boundary counterfactuals for every instance $\forall x \in D_{\text {correct }}^{c}$ with respect to every class $\forall j \in C \backslash c$. The output of this function is the set of generated boundary samples (denoted by $S$ ) and their distance to the original samples (denoted by $D$ ). In NEIGHBORHOODMODEL,

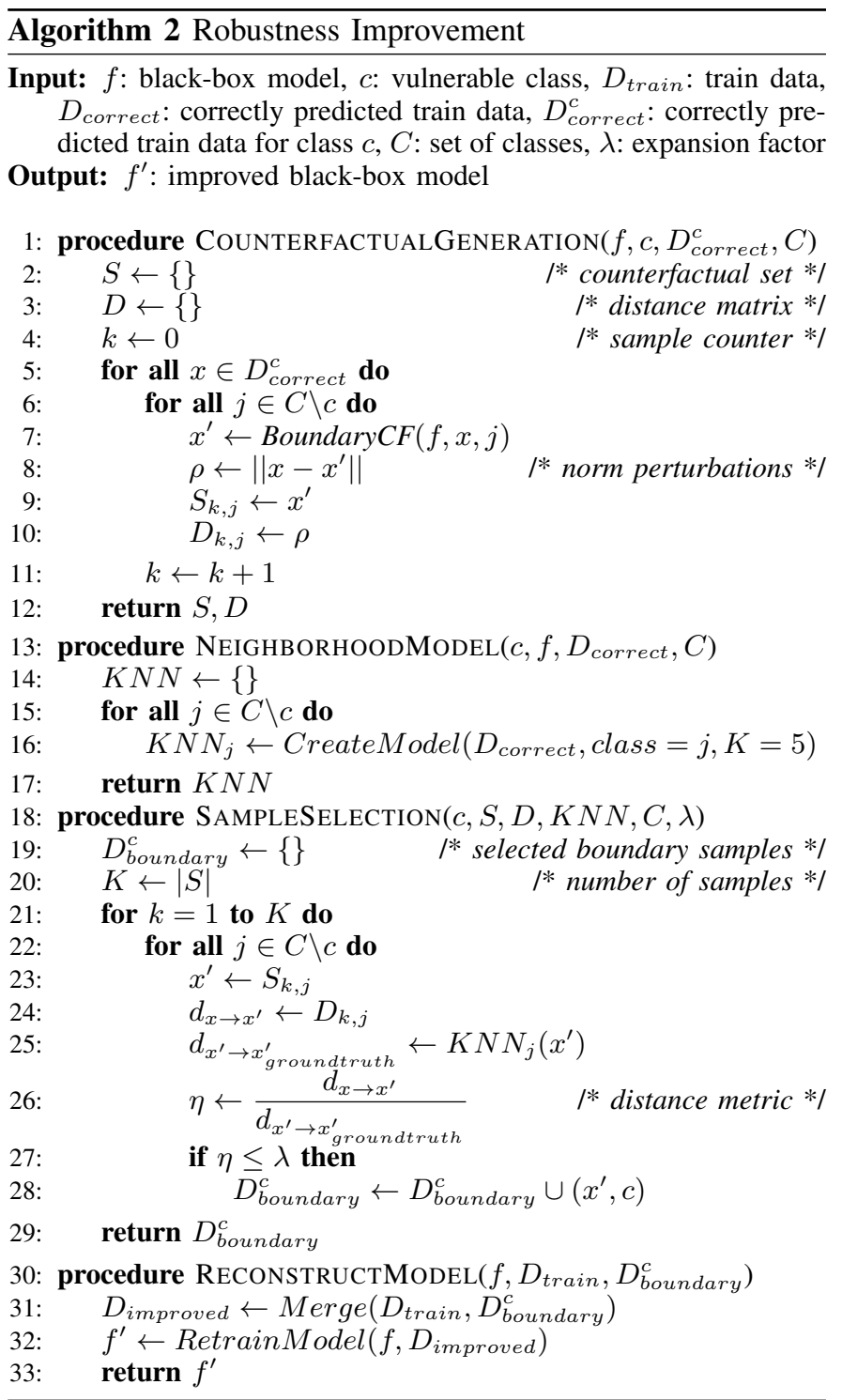

a k-nearest neighbor model for every class $\forall j \in C \backslash c$ based on $D_{\text {correct }}$ is created. The created models provide distance information for selecting representative samples in the subsequent procedure, i.e., SAMPLESELECTION. This function uses a distance metric $\eta$ to carefully choose the counterfactuals that are increasing the class margin of $c$ without compromising the accuracy of its adjacent classes $C \backslash c$. The metric is the ratio between the distance of the original instance to the counterfactual $\left(d_{x \rightarrow x^{\prime}}\right)$ and the average distance of the counterfactual to the same-class ground-truth data $\left(d_{x^{\prime} \rightarrow x_{\text {groundtruth }}^{\prime}}\right)$. We are interested in counterfactuals close to the original instances and far from the same-class ground-truth data. For example, among the illustrated counterfactuals in Figure 2 the boundary counterfactual generated for the data point from class 1 (denoted by Boundary CF - class 0 ) is a proper candidate. In this way, we are shifting the boundary without causing disturbance for the adjacent classes. We control the expansion of the decision boundary using parameter $\lambda \in \mathbb{R}$. 
TABLE I: Summary of information of the used data sets.

\begin{tabular}{lccc}
\hline Data set & \# Samples & \# Features & \# Classes \\
\hline Adult Income & 48842 & 14 & 2 \\
\hline Default of Credit Card Clients & 30000 & 23 & 2 \\
\hline COMPAS & 7214 & 11 & 2 \\
\hline German Credit & 1000 & 20 & 2 \\
\hline
\end{tabular}

The smaller the $\lambda$ is, the less interfering samples are selected that enlarge the margin. We can increase the $\lambda$ until we achieve a desired level of robustness without degrading the model's accuracy. We select the samples that meet the condition $\eta \leq \lambda$ and label them as the label of the vulnerable class $c$. Finally, in the ReconstructModel phase, the set of selected samples $D_{\text {boundary }}^{c}$ are merged with $D_{\text {train }}$, forming $D_{\text {improved }}$ that is used for creating an improved black-box model $f^{\prime}$.

\section{EXPERIMENTS AND DISCUSSION}

We demonstrate the efficacy of our approach using two types of evaluation: robustness analysis and robustness improvement. In Section IV-B, we analyze the robustness of various black-box models created for several data sets using class-wise and overall metrics. To demonstrate the efficacy of the proposed method in generating impactful perturbations, we compare the results with two baseline methods LowProFool [12] and DeepFool [8]. It should be mentiond that DeepFool is originally an effective attack algorithm for the image domain. However, due to the lack of proper baselines for tabular data, we customized it for tabular classifiers. In Section IV-C, we demonstrate the efficacy of the proposed technique in improving the robustness of black-box models against universal perturbations. The goal is to make a model robust without compromising its accuracy. We exhibit the performance of our approach through accuracy-robustness evaluation of improved models and assessing their vulnerability using baselines.

\section{A. Experimental Setup}

We mainly used standard data sets from the UCI Machine Learning Repository [15], except the COMPAS data set that can be found at [16]. Summary of information of the used data sets is reported in Table [1 Although our optimization approach can handle mixed-type data features, we only used numerical and ordinal features to make it compatible for baseline approaches. The selected features were standardized by removing the mean and scaled to unit variance.

We split the data sets into $80 \%$ train set and $20 \%$ test set. The train set was used for creating black-box and robustness improvement while the test set was used for measuring the accuracy and analyzing the model robustness. We created a Multi-layer Perceptron Neural Networks (NN) consisted of three hidden layers each with 500 neurons, Gradient Boosting Machines (GB) comprised of 100 estimators, and Support Vector Machines (SVM) as black-box classifiers.

For the NSGA-III optimization algorithm, we used the simulated binary bounded crossover operator with a percentage
TABLE II: Robustness analysis of the black-box models.

\begin{tabular}{lcccc}
\hline Data set & Metric & NN & GB & SVM \\
\hline \multirow{3}{*}{ Adult Income } & $R_{\text {overall }}$ & 0.143 & 0.068 & $\mathbf{0 . 1 5 5}$ \\
& $R_{\text {class }}^{0}$ & 0.712 & $\mathbf{1 . 8 3 7}$ & 0.713 \\
& $R_{\text {class }}^{1}$ & $\mathbf{1 . 4 0 5}$ & 0.544 & 1.402 \\
\hline \multirow{2}{*}{ Default of Credit } & $R_{\text {overall }}$ & 0.218 & 0.114 & $\mathbf{0 . 3 6 6}$ \\
Card Clients & $R_{\text {class }}^{0}$ & 3.228 & $\mathbf{4 . 1 1 8}$ & 2.919 \\
& $R_{\text {class }}^{1}$ & 0.310 & 0.243 & $\mathbf{0 . 3 4 3}$ \\
\hline \multirow{3}{*}{ COMPAS } & $R_{\text {overall }}$ & 0.215 & 0.145 & $\mathbf{0 . 4 0 4}$ \\
& $R_{\text {class }}^{0}$ & $\mathbf{0 . 4 8 4}$ & 0.254 & 0.309 \\
& $R_{\text {class }}^{1}$ & 2.066 & $\mathbf{3 . 9 4 0}$ & 3.239 \\
\multirow{3}{*}{ German Credit } & $R_{\text {overall }}$ & 0.550 & 0.160 & $\mathbf{0 . 8 5 2}$ \\
& $R_{\text {class }}^{0}$ & 1.829 & $\mathbf{3 . 6 4 1}$ & 3.216 \\
& $R_{\text {class }}^{1}$ & $\mathbf{0 . 5 4 7}$ & 0.275 & 0.311 \\
\hline
\end{tabular}

of $p c=60 \%$ and the polynomial mutation operator with a percentage of $\mathrm{pm}=40 \%$. The population size was determined as $n_{\text {population }}=100$ and the number of generations was set to $n_{\text {generation }}=50$. We used $\ell_{2}$-norm for measuring the norm of perturbations in our algorithm, which is identical to the norm used in baseline methods. For LowProFool and DeepFool, we set the number of iterations as $N_{\text {iter }}=10000$ and the perturbation growth factor as $\gamma=0.001$.

Our framework has been developed using Python programming language, and experiments were run on a system with Intel Core i7-8650U processor and 32GB of memory. A complete implementation of the method, including experiments, is available at: https://github.com/peymanrasouli/RobustML

\section{B. Robustness Analysis Evaluation}

We evaluated the overall and class-wise robustness of the created models using our proposed approach in Algorithm 1 We set the probability threshold as $p=0.5$. The results of this experiment are reported in TABLE II] According to the table, the most globally robust model for all data sets is SVM. This originates from the loss function of this model that focuses on creating a large margin decision boundary between distinct classes. It can be seen that our method has reflected this fact clearly. Among the evaluated black-boxes, GB has shown the most vulnerable model, however, for some specific classes, it demonstrates superior performance compared to $\mathbf{N N}$ and SVM. The class-wise robustness information is useful because it allows selecting a robust model concerning the sensitive class for the application, for example, the positive (spam) class in the spam detection system. In general, this information can be complementary to the accuracy performance to select an effective and reliable model for a given application.

In the following, we demonstrate the efficacy of our approach in generating impactful perturbations that can effortlessly deceive the model and remain imperceptible to the domain expert. Specifically, we benchmark our technique in fooling deep neural networks (NN) with two effective gradient-based algorithms, LowProFool [12] and DeepFool [8] regarding the metrics described below: 
TABLE III: Comparison of the efficacy of perturbations.

\begin{tabular}{lcccc}
\hline Data set & Metric & LowProFool & DeepFool & Proposed \\
\hline \multirow{4}{*}{ Adult Income } & $\rho$ & $0.073 \pm 0.06$ & $0.065 \pm 0.06$ & $\mathbf{0 . 0 6 5} \pm \mathbf{0 . 0 6}$ \\
& $\mathcal{P}$ & $\mathbf{0 . 5 7 6} \pm \mathbf{0 . 0 6}$ & $0.506 \pm 0.01$ & $0.504 \pm 0.00$ \\
& $D_{k n n}$ & $0.087 \pm 0.05$ & $0.085 \pm 0.05$ & $\mathbf{0 . 0 8 5} \pm \mathbf{0 . 0 5}$ \\
& $T_{\text {exe }}$ & $11.88 \pm 1.10$ & $\mathbf{0 . 1 4 0} \pm \mathbf{0 . 1 8}$ & $2.828 \pm 0.15$ \\
\hline \multirow{3}{*}{ Default of Credit } & $\rho$ & $0.296 \pm 0.31$ & $\mathbf{0 . 1 3 8} \pm \mathbf{0 . 0 7}$ & $0.154 \pm 0.08$ \\
Card Clients & $\mathcal{P}$ & $\mathbf{0 . 6 1 9} \pm \mathbf{0 . 2 0}$ & $0.500 \pm 0.01$ & $0.507 \pm 0.01$ \\
& $D_{k n n}$ & $0.331 \pm 0.21$ & $0.223 \pm 0.07$ & $\mathbf{0 . 2 1 8} \pm \mathbf{0 . 0 8}$ \\
& $T_{\text {exe }}$ & $12.19 \pm 1.30$ & $\mathbf{0 . 3 6 9} \pm \mathbf{0 . 2 6}$ & $3.279 \pm 0.31$ \\
& $\rho$ & $0.136 \pm 0.11$ & $0.134 \pm 0.11$ & $\mathbf{0 . 1 3 0} \pm \mathbf{0 . 1 0}$ \\
COMPAS & $\mathcal{P}$ & $\mathbf{0 . 5 1 2} \pm \mathbf{0 . 0 1}$ & $0.501 \pm 0.00$ & $0.502 \pm 0.00$ \\
& $D_{k n n}$ & $0.074 \pm 0.06$ & $0.073 \pm 0.06$ & $\mathbf{0 . 0 6 6} \pm \mathbf{0 . 0 5}$ \\
& $T_{\text {exe }}$ & $12.21 \pm 1.43$ & $\mathbf{0 . 1 8 8} \pm \mathbf{0 . 1 6}$ & $3.349 \pm 0.04$ \\
\hline \multirow{3}{*}{ German Credit } & $\rho$ & $0.313 \pm 0.12$ & $\mathbf{0 . 3 1 1} \pm \mathbf{0 . 1 2}$ & $0.343 \pm 0.13$ \\
& $\mathcal{P}$ & $0.505 \pm 0.01$ & $0.504 \pm 0.01$ & $\mathbf{0 . 5 1 5} \pm \mathbf{0 . 0 2}$ \\
& $D_{k n n}$ & $0.634 \pm 0.15$ & $0.633 \pm 0.15$ & $\mathbf{0 . 6 2 5} \pm \mathbf{0 . 1 6}$ \\
& $T_{\text {exe }}$ & $14.02 \pm 0.01$ & $\mathbf{0 . 6 0 5} \pm \mathbf{0 . 2 3}$ & $3.360 \pm 0.03$ \\
\hline
\end{tabular}

- Norm Perturbations $(\rho)$ : this metric measures the average $\ell_{2}$-norm perturbations added to the original inputs. It indicates the effort required to cross the decision boundary between a pair of classes to achieve the desired outcome from the model. Smaller values refer to data points that seem normal from the expert's viewpoint.

- Prediction Probability $(\mathcal{P})$ : this metric measures the average prediction probability of the evasive data points. Since prediction probability reflects the certainty of the model's decision, instances with high $\mathcal{P}$ can bypass the monitoring process and appear as natural inputs.

- Distance to K-Nearest Neighbors $\left(D_{k n n}\right)$ : this metric measures the average distance of evasive samples to their $k$-nearest same-class ground-truth instances in the training data. Smaller $D_{K N N}$ values indicate more imperceptible data points that correspond to the distribution of the targeted class.

- Computational Complexity $\left(T_{\text {exe }}\right)$ : this metric measures the average amount of time to generate an evasive sample. The results are shown in seconds where the lower values indicate the more efficient algorithms.

The results of this evaluation are presented in TABLE III] Concerning the norm of perturbations. i.e., $\rho$ metric, our approach demonstrates comparable performance with the baseline methods. It should be noted that LowProFool and DeepFool are model-specific, gradient-based methods that can effectively generate near-optimal solutions through accessing the model internals. Yet, our model-agnostic approach has achieved comparable results within a reasonable amount of time. Regrading the prediction probability, i.e., $\mathcal{P}$, Low ProFool has shown the best performance. This originates from the main purpose of this approach which is generating imperceptible perturbations based on the domain knowledge, leading to natural instances. The generated data points by our method are the closest to the ground-truth data of the targeted class (minimum values for $D_{k n n}$ ). It is a desirable property to create minimally modified instances that simultaneously resemble the samples of the attacked class. Computational complexity is a critical aspect of robustness analysis techniques. On average, our algorithm takes 3.33 seconds to create evasive data points while LowProFool and DeepFool take 11.69 and 0.3 seconds, respectively. This is comparable for all data sets since they share a similar number of features. The baseline approaches follow a bottom-up approach as they iteratively increase perturbations until they find a sample with a different label, leading to execution termination. In contrast, our algorithm follows a top-down procedure that refines the solution during determined iterations. Therefore, specifying a perturbation threshold as a termination condition for our approach results in even a lower time complexity. It is noteworthy that our method is model-agnostic, as it only relies on the model's output and generates counterfactuals irrespective of the model's complexity, resulting in an efficient performance for various models.

\section{Robustness Improvement Evaluation}

In this section, we evaluate the performance of our proposed technique in improving the robustness of black-box classifiers. We apply Algorithm 2 on the original model $f$ and the corresponding train data $\left(D_{\text {train }}\right)$; afterward, we measure the enhancement of the improved model $f^{\prime}$ using the test data $\left(D_{\text {test }}\right)$. It is noteworthy that the test set is remained untouched throughout the refinement process to prevent the distribution shift. We improve the robustness of vulnerable classes that are identified by Algorithm 1 . An appropriate range for the expansion factor (i.e., $\lambda$ ) is $[0,1]$. Samples selected within this range are likely to increase the robustness of the model without degrading its accuracy. To find the best setting for every pair of data set and model, we repeated the Algorithm 2 with different values of $\lambda \in[0,1]$ and selected a model that had the highest accuracy and robustness performance.

The main goal of our approach is to enhance the robustness of a vulnerable class without negatively affecting the model's accuracy. As the first experiment, we apply Algorithm 2 to various black-box models and report the accuracy and robustness of the original and improved models in TABLE IV] Based on the evaluation results, we mention key observations regarding the performance of our algorithm. First, our algorithm has significantly preserved the accuracy of the original models because of the proper sample selection approach. Second, it enhanced the robustness of the vulnerable class, which accordingly causes a decline in the robustness of the strong class due to balancing the class margins. Third, compared to the original models, the robustness of classes in the improved models are closer to $R_{\text {class }} \simeq 1.0$, implying a globally robust classifier that has classes with a similar margin to the decision boundary. Fourth, among the studied black-boxes, SVM model has shown to be more rigid concerning boundary shifting, while NN and GB models allow easier refinement.

A reliable metric for robustness improvement is to measure the required perturbations for misclassifying the samples of the vulnerable class. The magnitude of perturbations is determined by $\varepsilon \in \mathbb{R}$. Given a specific value for $\varepsilon$, we can measure 
TABLE IV: Accuracy-robustness evaluation: original models (withe columns) vs. improved models (gray columns).

\begin{tabular}{lccccccc}
\hline Data set & Metric & \multicolumn{2}{c}{ NN } & \multicolumn{2}{c}{ GB } & \multicolumn{2}{c}{ SVM } \\
\hline \multirow{4}{*}{ Adult Income } & Precision & 0.821 & 0.824 & 0.841 & 0.841 & 0.823 & 0.824 \\
& Recall & 0.828 & 0.831 & 0.846 & 0.847 & 0.829 & 0.829 \\
& $R_{\text {class }}^{0}$ & 0.711 & 0.934 & 1.890 & 1.107 & 0.695 & 0.720 \\
& $R_{\text {class }}^{1}$ & 1.406 & 1.071 & 0.529 & 0.903 & 1.438 & 1.389 \\
\hline \multirow{3}{*}{ Default of Credit } & Precision & 0.800 & 0.800 & 0.802 & 0.801 & 0.799 & 0.802 \\
Card Clients & Recall & 0.818 & 0.813 & 0.820 & 0.819 & 0.817 & 0.820 \\
& $R_{\text {class }}^{0}$ & 3.047 & 1.150 & 3.971 & 1.213 & 2.914 & 2.252 \\
& $R_{\text {class }}^{1}$ & 0.328 & 0.870 & 0.252 & 0.824 & 0.343 & 0.444 \\
\hline \multirow{4}{*}{ COMPAS } & Precision & 0.795 & 0.800 & 0.802 & 0.804 & 0.781 & 0.781 \\
& Recall & 0.805 & 0.809 & 0.811 & 0.812 & 0.787 & 0.788 \\
& $R_{\text {class }}^{0}$ & 0.511 & 0.565 & 0.258 & 0.446 & 0.310 & 0.334 \\
& $R_{\text {class }}^{1}$ & 1.957 & 1.769 & 3.869 & 2.241 & 3.230 & 2.994 \\
\hline \multirow{4}{*}{ German Credit } & Precision & 0.746 & 0.769 & 0.770 & 0.746 & 0.767 & 0.782 \\
& Recall & 0.760 & 0.780 & 0.780 & 0.760 & 0.765 & 0.785 \\
& $R_{\text {class }}^{0}$ & 2.073 & 1.634 & 4.068 & 1.371 & 3.235 & 2.743 \\
& $R_{\text {class }}^{1}$ & 0.482 & 0.612 & 0.246 & 0.729 & 0.309 & 0.365 \\
\hline
\end{tabular}

the success rate of an algorithm in generating samples that successfully deceive the classifier and lead to misprediction. Normally, increasing the robustness of a model should decrease a method's success rate given a fixed value for $\varepsilon$. We measure the success rate of LowProFool and DeepFool with respect to varying values of $\varepsilon$ on an original neural network $\left(\mathbf{N N}_{\text {original }}\right)$ and its improved counterpart $\left(\mathbf{N N}_{\text {improved }}\right)$ for Adult data set. According to the illustrated results in Figure 3 the improved model is more robust against perturbations, which originates from the enlargement of class margin that prevents minor noises evade the model's behavior.

The devised robustness improvement method (Algorithm 2) is computationally efficient. The major computational burden is caused by COUNTERFACTUALGENERATION that requires $O\left(M N^{2}\right)$ computations for generating a boundary counterfactual, where $M$ is the number of objectives and $N$ is the population size. On the other hand, NEIGHBORHOODMODEL can create an advanced nearest neighborhood model like KD-Tree with only $O(D N \log (N))$ computations, where $D$ is the dimension of features, and $N$ is the number of samples. The SAMPLESELECTION procedure is very cost-efficient as it only performs neighborhood query and lightweight operations. Eventually, the burden of RECONSTRUCTMODEL significantly depends on the complexity of the studied black-box model.

\section{CONCLUSIONS AND FUtURE WORKS}

In this paper, we uncovered the utility of counterfactual explanations in analyzing and improving the robustness of ML models without compromising their accuracy. Accordingly, we proposed an algorithm to measure and compare the global and local robustness of black-box classifiers against universal perturbations in the tabular setting. Furthermore, we improved the robustness of the vulnerable classes via a novel decision boundary modification technique. Several experiments on various data sets and models demonstrated the efficacy of our framework in identifying and enhancing

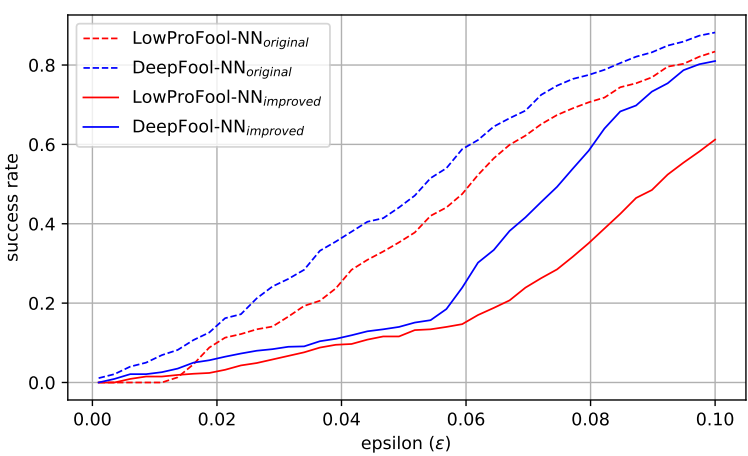

Fig. 3: Success rate: original vs. improved model.

the model's vulnerabilities. In future works, we explore the potential benefits of counterfactuals for other aspects of model improvement, such as fairness and accuracy.

\section{REFERENCES}

[1] S. Qiu, Q. Liu, S. Zhou, and C. Wu, "Review of artificial intelligence adversarial attack and defense technologies," Applied Sciences, vol. 9, no. 5, p. 909, 2019.

[2] S. Bhambri, S. Muku, A. Tulasi, and A. B. Buduru, "A survey of black-box adversarial attacks on computer vision models," arXiv preprint arXiv:1912.01667, 2019.

[3] T. Bai, J. Luo, J. Zhao, B. Wen, and Q. Wang, "Recent advances in adversarial training for adversarial robustness," arXiv preprint arXiv:2102.01356, 2021.

[4] C. Song, Z. Wang, and H. Li, "Feedback learning for improving the robustness of neural networks," in 2019 18th IEEE International Conference On Machine Learning And Applications (ICMLA). IEEE, 2019, pp. 686-693.

[5] G. F. Elsayed, D. Krishnan, H. Mobahi, K. Regan, and S. Bengio, "Large margin deep networks for classification," arXiv preprint arXiv:1803.05598, 2018.

[6] Y. Guo and C. Zhang, "Recent advances in large margin learning," arXiv preprint arXiv:2103.13598, 2021.

[7] P. Li, J. Yi, B. Zhou, and L. Zhang, "Improving the robustness of deep neural networks via adversarial training with triplet loss," arXiv preprint arXiv:1905.11713, 2019.

[8] S.-M. Moosavi-Dezfooli, A. Fawzi, and P. Frossard, "Deepfool: a simple and accurate method to fool deep neural networks," in Proceedings of the IEEE conference on computer vision and pattern recognition, 2016 , pp. 2574-2582.

[9] L. Ma and L. Liang, "Increasing-margin adversarial (ima) training to improve adversarial robustness of neural networks," arXiv preprint arXiv:2005.09147, 2020.

[10] F. Cartella, O. Anunciacao, Y. Funabiki, D. Yamaguchi, T. Akishita, and O. Elshocht, "Adversarial attacks for tabular data: Application to fraud detection and imbalanced data," arXiv preprint arXiv:2101.08030, 2021.

[11] S. Wachter, B. Mittelstadt, and C. Russell, "Counterfactual explanations without opening the black box: Automated decisions and the gdpr,' Harv. JL \& Tech., vol. 31, p. 841, 2017.

[12] V. Ballet, X. Renard, J. Aigrain, T. Laugel, P. Frossard, and M. Detyniecki, "Imperceptible adversarial attacks on tabular data," arXiv preprint arXiv:1911.03274, 2019.

[13] W. He, B. Li, and D. Song, "Decision boundary analysis of adversarial examples," in International Conference on Learning Representations, 2018.

[14] K. Deb and H. Jain, "An evolutionary many-objective optimization algorithm using reference-point-based nondominated sorting approach, part i: solving problems with box constraints," IEEE transactions on evolutionary computation, vol. 18, no. 4, pp. 577-601, 2013.

[15] D. Dua and C. Graff, "UCI machine learning repository," 2017. [Online]. Available: http://archive.ics.uci.edu/ml

[16] ProPublica, "Compas data set," 2017. [Online]. Available: https: //www.kaggle.com/danofer/compass 